\title{
Structural mapping in statistical word problems: A relational reasoning approach to Bayesian inference
}

\author{
Eric D. Johnson ${ }^{1,2}$ - Elisabet Tubau ${ }^{1,2}$
}

Published online: 27 September 2016

(C) Psychonomic Society, Inc. 2016

\begin{abstract}
Presenting natural frequencies facilitates Bayesian inferences relative to using percentages. Nevertheless, many people, including highly educated and skilled reasoners, still fail to provide Bayesian responses to these computationally simple problems. We show that the complexity of relational reasoning (e.g., the structural mapping between the presented and requested relations) can help explain the remaining difficulties. With a non-Bayesian inference that required identical arithmetic but afforded a more direct structural mapping, performance was universally high. Furthermore, reducing the relational demands of the task through questions that directed reasoners to use the presented statistics, as compared with questions that prompted the representation of a second, similar sample, also significantly improved reasoning. Distinct error patterns were also observed between these presented- and similar-sample scenarios, which suggested differences in relational-reasoning strategies. On the other hand, while higher numeracy was associated with better Bayesian reasoning, higher-numerate reasoners were not immune to the relational complexity of the task. Together, these findings validate the relational-reasoning view of Bayesian problem solving and highlight the importance of considering not only the presented task structure, but also the complexity of the structural alignment between the presented and requested relations.
\end{abstract}

Eric D. Johnson

eric.johnson@ub.edu

1 Department of Basic Psychology, University of Barcelona, Barcelona, Spain

2 IR3C, University of Barcelona, Barcelona, Spain
Keywords Bayesian inference $\cdot$ Natural frequencies · Relational reasoning $\cdot$ Numeracy $\cdot$ Question form $\cdot$ Structural mapping

Educated adults are notoriously poor Bayesian reasoners with explicit numerical information (for a recent review, see Johnson \& Tubau, 2015). Although presenting statistical information as natural frequencies is the most widely agreed facilitator of Bayesian inferences (Gigerenzer \& Hoffrage, 1995), many reasoners still fail to solve these problems correctly. Why does Bayesian-like reasoning remain so difficult, even after providing reasoners with natural frequencies? In this article, we address this question by viewing Bayesian reasoning as a case of relational reasoning, which requires the comparison of role-based structural relations across multiple mental representations (Halford, Wilson, \& Phillips, 1998, 2010; Holyoak, 2012).

The medical-diagnosis problem represents a typical Bayesian reasoning task (Table 1). In this problem, information is presented regarding the base rate of having a disease and the likelihood of testing positive with a diagnostic test. As is illustrated in Fig. 1, this information can be represented structurally as a series of nested sets. From this information, the standard Bayesian question- $(H \mid D)$ - asks reasoners to compute the expected number of infected people (the hypothesis, $H$ ) given a positive test result (the data, $D$ ). The solution, given natural frequencies, can be represented with the Bayesian equation:

$$
\begin{aligned}
(H \mid D) & =\frac{(H \& D)}{(D)}=\frac{(H \& D)}{(H \& D)+(\neg H \& D)}=\frac{16}{16+24} \\
& =\text { "16 out of } 40 . "
\end{aligned}
$$

Intense theoretical debate persists regarding the mechanism through which natural frequencies facilitate performance over, 


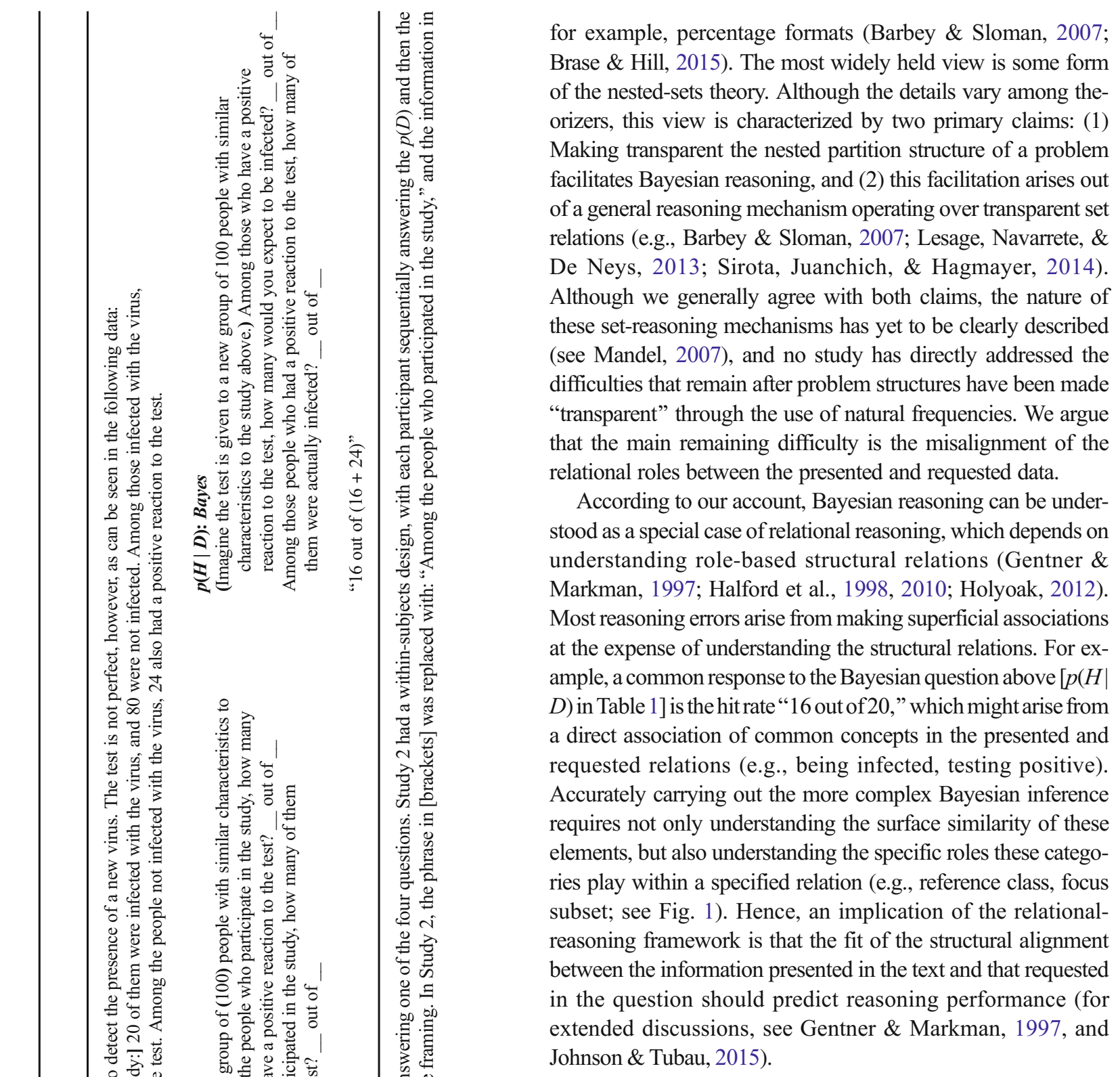

\section{Alignment hypothesis}

From the perspective of relational reasoning, solving the Bayesian question would be difficult due to the change in the relational role of the subsets required to compute the reference class. That is, the new reference class- $(D$, all positive tests $)$ must be computed from the previously presented focal subsets ( $H \& D=$ positive among infected and $\neg H \& D=$ positive among not infected), from which the new focal subset $(H \& D)$ is selected (see Fig. 1). Some studies have suggested that people do not understand that they must use both the focal $(H \& D)$ and alternative $(\neg H \& D)$ hypotheses to compute the relevant reference class of the posterior ratio $(D)$, where people tend to neglect the alternative hypothesis (see Evans et al., 2000; Girotto \& Gonzalez, 2001; Krynski \& Tenenbaum, 2007). On the basis of the 
(A)

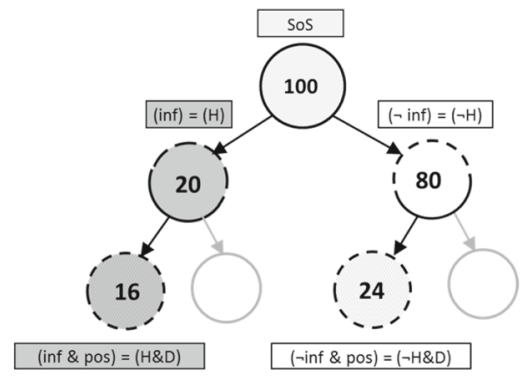

(B)

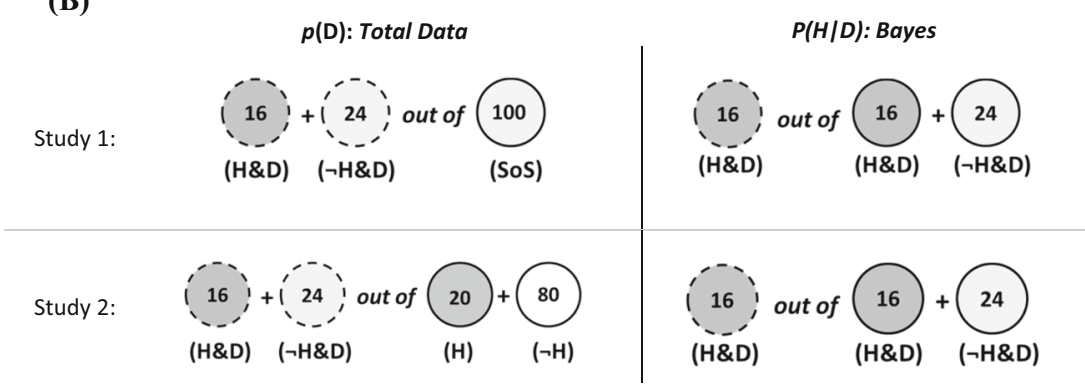

Fig. 1 The information in Table 1, represented structurally as a series of nested set relations. (A) The structure of the presented information and its relations to the Bayesian equation. (B) The requested relations needed to solve the $p(D)$ and $p(H \mid D)$ questions in Studies 1 and 2. The relational roles played by the different elements (e.g., $H, D$ ) are illustrated with solid lines (= reference class) and dotted lines (= focal subset). Note that $H$ and

relational-reasoning approach, we hypothesized that the difficulty computing $(D)$ is specific to its different role in the Bayesian relation (as reference class), relative to its role in the presented relations (as a pair of focal subsets), rather than arising from the computation of $(D)$ itself (alignment hypothesis).

To test this hypothesis, we created a non-Bayesian condition - the total-data question, $p(D)$ - that required arithmetic identical to that in the Bayesian question, but where the elements (e.g., positive tests) played the same relational roles as in the presented data (Table 1). In this condition, the required reference class was the superordinate set $(\mathrm{SoS})$ of the problem, and the new focal subset required summing of the two initial focal subsets (the frequencies corresponding to $H \& D+$ $\neg H \& D$ ). Accordingly, the $p(D)$ question also provided a control of participants' ability to select the particular subsets needed to compute the posterior reference class. The required computations are illustrated in the equation:

$$
\begin{aligned}
(D) & =\frac{(D)}{(S o S)}=\frac{(H \& D)+(\neg H \& D)}{(S o S)}=\frac{16+24}{100} \\
& =\text { "40 out of 100." }
\end{aligned}
$$

\section{Sample-type hypothesis}

In addition to the mapping complexity between the presented and requested relations, typical Bayesian tasks request $\neg H$ both play roles in the presented data. In $p(D)$, the relational roles remain the same between the presented and requested data, but they are misaligned with the $p(H \mid D)$ question. $H=$ infected; $\neg H=$ not infected; $H \& D=$ infected and test positive; $\neg H \& D=$ not infected and test positive; $\mathrm{SoS}=$ superordinate set

participants to reason over a new, unspecified sample similar to the one presented (see Table 1, "similar-sample questions"). This requires the reasoner to infer the new statistics based on the presented statistics prior to carrying out the Bayesian inference. A series of informal observations in pilot studies in our lab suggested that simply asking participants to base their answer on the presented data greatly facilitated Bayesian responses. From the relational-reasoning account, a question prompting a reasoner to imagine a similar sample would increase processing demands due to the need to maintain different samples (i.e., the presented sample and the imagined similar sample) to infer the corresponding statistics. Likewise, instructing participants to base their answer on the presented data would facilitate exact Bayesian responses by eliminating the need to construct a second representation and perform the corresponding mapping. We tested this sample-type hypothesis by directly instructing participants to reason over the sample of individuals presented in the text (presentedsample question) or over a new, similar sample of individuals (similar-sample question; see Table 1).

Alternatively, asking them to imagine a similar sample might lead participants to base responses on an approximation or a compute-then-adjust strategy to accommodate the "similar" sample. In this case, more approximate estimations should be observed in the responses to both Bayesian and non-Bayesian questions. However, if, as we suggested above, imagining a similar sample hampered performance due to increased relational reasoning being demanded, this effect should be stronger for the more- 
complex Bayesian question. To distinguish between these possibilities, error analyses were used to gain insight into the processing strategies that individuals were engaging. Given that these hypothesized effects might also be moderated by individual skills, we measured participants' levels of numeracy, which has commonly been observed to influence probabilistic-reasoning performance (e.g., Johnson \& Tubau, 2013; McNair \& Feeney, 2015).

\section{Study 1}

In Study 1, we used a between-participants design to test the sample-type hypothesis - that Bayesian performance can be improved by instructing reasoners to use the data presented in the problem rather than imagining a new, similar sample - and the alignment hypothesis - that even when identical numerical computations were requested from identically presented data, a specific difficulty would be observed with the Bayesian logic.

\section{Method and materials}

Due to an a priori power calculation to detect a medium-sized effect $(w=0.4)$, we aimed to include at least 60 participants for each condition (a conservative estimate, considering previous research; e.g., McNair \& Feeney, 2015; Sirota et al., 2014; Sirota, Kostovičová, \& Vallée-Tourangeau, 2015). The participants were 319 undergraduates from the University of Barcelona who had yet to receive instruction in Bayesian reasoning. Informed consent was collected, and students received course credit for their participation. All participants received the virus test scenario and either the $p(D)$ or the $p(H \mid D)$ question, referring to either an imagined similar sample or the presented sample shown in Table 1. Following this, participants completed the 11-item Lipkus, Samsa, and Rimer (2001) numeracy scale. Calculators were not allowed.

(A)

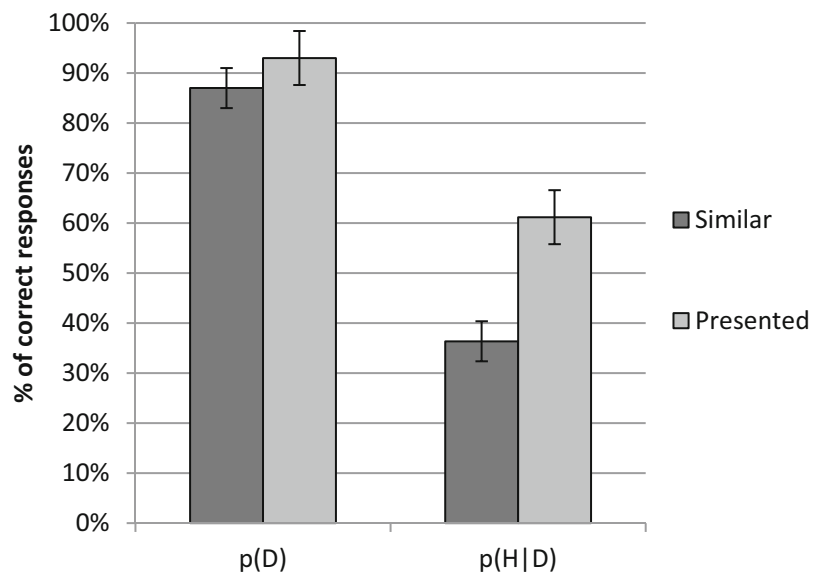

\section{Results}

Nine participants were removed from the study for indicating that they had previously seen this type of problem or for not completing the task as requested. Results are therefore reported for the remaining 310 participants (mean age $=21.4$ years, $S D=1.6$ ). Their mean numeracy score was 8.65 (median $=9$, range $=3-11, S D=1.86$ ).

Reasoning accuracy Global results are summarized in Fig. 2. Only exact Bayesian answers were counted as correct. A logistic regression was performed using a dichotomous coding of responses (correct, incorrect) as the dependent variable, with the question $[p(H \mid D), p(D)]$, sample type (similar, presented), and continuous numeracy score entered as predictors.

The results revealed a significant main effect of sample type, $\chi^{2}(1)=12.36, p<.001, e^{\beta}=2.90,95 \%$ confidence interval (C.I.) $=1.60-5.24$, showing facilitated performance when the question directed reasoners to use the specific data presented in the problem. There was also a significant effect of question, $\chi^{2}(1)=50.92, p<.001, e^{\beta}=11.22,95 \%$ C.I. $=$ 5.77-21.79, with ceiling performance observed on the $p(D)$ question. Finally, as is illustrated in Fig. 3A, a significant effect of numeracy was also observed, $\chi^{2}(1)=14.65, p<$ $.001, e^{\beta}=1.37,95 \%$ C.I. $=1.17-1.61$, with higher-numerate individuals being better with both the presented and similar scenarios. No significant interactions emerged (all $p \mathrm{~s}>.20$ ).

Error analysis Table 2 reports the observed errors on the $p(H$ $D$ ) question. Errors in the similar- sample condition were widely distributed, however, over half of these errors contained the superordinate value 100 as the reference class. In the presented-sample condition, less than a third of all errors contained the value 100 . This difference in responses containing " 100 " was significant, $\chi^{2}(N=153)=11.47, p<$ $.001, \varphi=.27$. Furthermore, in the similar-sample scenario

(B)

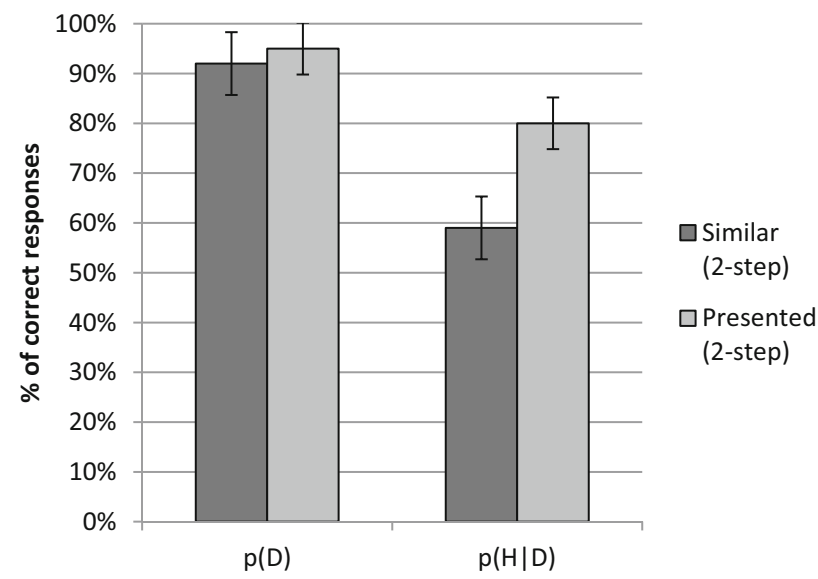

Fig. 2 Proportions of correct responses with similar-sample versus presented-sample questions, with the alignment manipulation, $p(D)$ versus $p(H \mid D)$, done (A) between subjects in Study 1 and (B) within subjects as a two-step manipulation in Study 2. Error bars indicate standard errors 
(A)

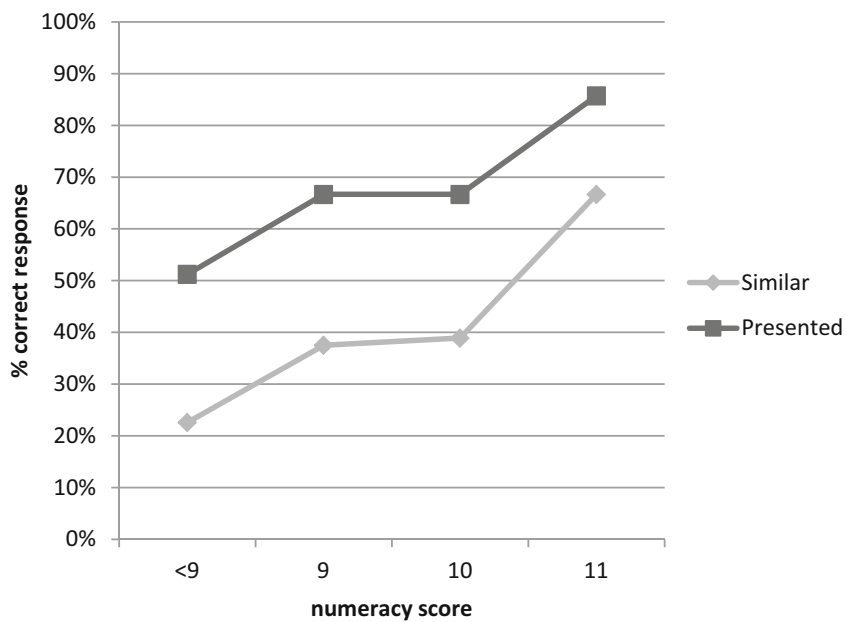

(B)

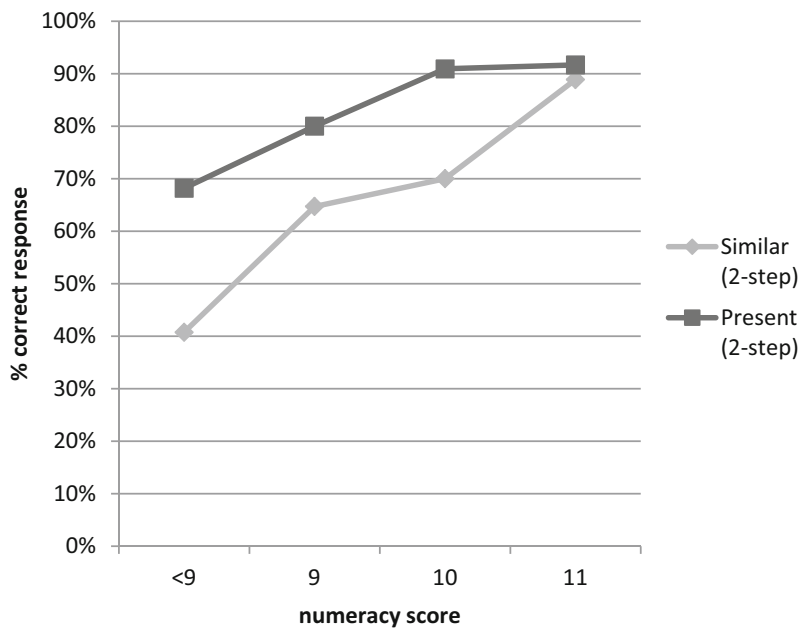

Fig. 3 Performance on the Bayesian $p(H \mid D)$ question according to numeracy score: (A) in Study 1, and (B) in Study 2 with a two-step manipulation

only a few hit-rate-only responses were observed, whereas this was the most frequent error in the presented-sample scenario, $\chi^{2}(N=153)=5.91, p=.015, \varphi=.20($ see Table 2$)$.

\section{Discussion}

The results of this study supported the two primary hypotheses. First, we obtained an alignment effect: significantly better performance when the relational roles in the presented and requested set-subset data were aligned. Second, we observed a sample-type effect: improved performance from instructing participants to reason over the data presented in the problem (presented-sample question) rather than a similar sample of individuals (similar-sample question). The ceiling performance with the $p(D)$ question, requiring the same computations as the more complex $p(H \mid D)$ question, indicates that difficulties determining the Bayesian reference class (the total set of positive tests) are specific to its particular role in the posterior ratio. Error analyses suggested that the lower performance in the similar-sample scenario was not caused simply by more approximate reasoning strategies, since this would have also reduced exact responses in the $p(D)$ question. Nevertheless, a large number of responses to the similar- sample scenario contained the value 100 . The presence of this value in the both presented data and the similar-sample scenario questions (see Table 1) could have both hampered the difficult question and enhanced the easier one. In Study 2, we attempted to solve this issue.

\section{Study 2}

In this study, we provided a more stringent test of the sampletype and alignment hypotheses. As we commented above, the inclusion of " 100 " in both the data and the question of the similar-sample scenario may have both interfered with selection of the relevant information for the $p(H \mid D)$ question and facilitated performance for $p(D)$, which together would undermine the observed alignment effect, in addition to weakening the sample-type effect. Therefore, in this study we removed all references to the superordinate set value 100 from the problem text and question. This both removed irrelevant information from the $p(H \mid D)$ problem and added an additional arithmetic step to the $p(D)$ question (Fig. 2). We also adopted a two-step design in which participants were sequentially presented with

Table 2 Percentages of responses observed with the posterior $p(H \mid D)$ question in Studies (St.) 1 and 2

\begin{tabular}{lllllllll}
\hline St. & Sample Type & $(n)$ & $\begin{array}{l}\text { Bayes } \\
(16 / 40)\end{array}$ & $\begin{array}{l}\text { Pre-Bayes } \\
(20 / 40)\end{array}$ & $\begin{array}{l}\text { HR } \\
(16 / 20)\end{array}$ & $\begin{array}{l}\text { BR } \\
(20 / 100)\end{array}$ & $\begin{array}{l}\text { Joint } \\
(16 / 100)\end{array}$ & $\begin{array}{l}\text { 100-othr } \\
(\mathrm{xx} / 100)\end{array}$ \\
\hline 1 & Similar & $(69)$ & $\mathbf{3 6}$ & 10 & 3 & 12 & 6 & 17 \\
& Present & $(84)$ & $\mathbf{6 1}$ & 4 & 14 & 2 & 2 & 7 \\
2 & Similar (2-stp) & $(63)$ & $\mathbf{5 9}$ & 8 & 8 & 3 & 6 & 16 \\
& Present (2-stp) & $(60)$ & $\mathbf{8 0}$ & 2 & 12 & 0 & 2 & 0 \\
\hline
\end{tabular}

Bayes = exact Bayesian solution $(H \& D$ out of $D)$; Pre-Bayes = correct denominator but selected all of the infected as numerator $(H$ out of $D)$; HR $=$ hit rate $(H \& D$ out of $H) ; \mathrm{BR}=$ base rate $(H$ out of 100$)$; Joint $=$ joint occurrence $(H \& D$ out of 100$)$; 100 -othr $=$ other numerators paired with the superordinate set as denominator (xx out of 100) 
the $p(D)$ and $p(H \mid D)$ questions as a within-subjects manipulation.

We again expected near-ceiling performance with the initial $p(D)$ question. We hypothesized that these manipulations would facilitate Bayesian responding in both the similar- and presented-sample conditions. A significant sample-type effect under these highly facilitatory conditions would be clear evidence that reasoning over similar samples requires additional processing, in comparison to reasoning over the presented samples.

\section{Method and materials}

A new sample of 123 undergraduate students (mean age $=$ 21.2 years, $S D=1.4$ ) completed the $p(D)$ question followed by $p(H \mid D)$, both with either the presented-sample or the similar-sample framing, and also the numeracy scale. The alignment manipulation was therefore done within participants, whereas the sample-type manipulation took place between participants. All explicit mentions of "100" were removed from the problem, as is indicated in Table 1.

\section{Results}

Reasoning accuracy Global results are shown in Fig. 2B. The mean numeracy score was 8.76 (median $=9$, range $=3-11, S D$ $=1.71)$. With the $p(D)$ question, ceiling performance was again observed for both sample-type scenarios, and a logistic regression run on this first step showed no differences between sample type and numeracy scores, and no interaction, all $\chi^{2} \mathrm{~s}(1)<1.5, p \mathrm{~s}>.25$. In contrast, a logistic regression on the $p(H \mid D)$ responses revealed significant main effects of both sample type, $\chi^{2}(1)=6.91, p=.009, e^{\beta}=.31,95 \%$ C.I. $=.13-.74$, and numeracy, $\chi^{2}(1)=11.27, p=.001, e^{\beta}=1.54$, $95 \%$ C.I. $=1.20-1.98$ (Fig. $3 \mathrm{~B}$ ). The interaction was not significant, $\chi^{2}(1)<1, p>.75$.

A within-subjects McNemar test also confirmed alignment effects with both the presented- sample, McNemar $\chi^{2}(N=60)$ $=9.0, p=.004, \varphi=.46$, and the similar-sample, McNemar $\chi^{2}(N=63)=17.649, p<.001, \varphi=.11$, scenarios. To further explore this effect according to numeracy level, a median split was used to separate participants into higher- and lowernumeracy groups. For lower-numerate individuals, a significant alignment effect was observed for both the similar-sample, McNemar $\chi^{2}(N=27)=13.00, p<.001, \varphi=.29$, and the presented-sample, $\operatorname{McNemar} \chi^{2}(N=22)=6.00, p=.031, \varphi=$ .32 , scenarios. For higher-numerate reasoners, the alignment effect just reached significance with the similar-sample scenario, McNemar $\chi^{2}(N=36)=5.33, p=.039, \varphi=.15$; however, it disappeared in the presented-sample condition, $\operatorname{McNemar} \chi^{2}(N=38)=3.00, p=.25$.
Error analysis Globally, the most common errors paralleled those found in the previous study (Table 2). In particular, although errors in the similar-sample condition were widely distributed, the use of 100 as the reference class was still more frequent than with the presented-sample scenario, $\chi^{2}(N=123)$ $=6.55, p=.010, \varphi=.23$. Also as before, most of the errors in the presented-sample scenario corresponded to the hit rate (58\% of errors, vs. $14 \%$ of the errors in the similar-sample condition), $\chi^{2}(N=38)=5.81, p=.016, \varphi=.39$.

\section{Discussion}

The present study provides additional support for the sampletype effect. Even with two-step questions that guided reasoners through the necessary computations, instructing reasoners to use the presented data still facilitated Bayesian responses, as compared with similar samples. As we discuss below, the error analysis also suggested that using the presented sample enhanced the mapping between the presented and requested relations. Nevertheless, an alignment effect was still observed for individuals lower in numeracy. Confirming previous findings, almost all participants in both conditions could use the presented information to accurately compute the total number of positive tests using the hit rate and the falsepositive rate, but many of them still failed to use this computation as the reference class for the Bayesian ratio. Together, this indicates a specific difficulty with the structural mapping required to supply the Bayesian response.

\section{General discussion}

In these studies, we asked why Bayesian-like reasoning remains so difficult, even after clarifying the nested-set structure of the problems with natural frequencies. According to our account, statistical word problems require a type of relational reasoning, and therefore performance should be influenced by the relational complexity of the task. Solving the Bayesian $p(H \mid D)$ question requires realizing that the relational roles of the specific subsets presented in the text are changed in the question. With the non-Bayesian $p(D)$ question, on the other hand, the relational roles of the focal and reference classes are maintained between the presented and the requested information, providing a more direct structural mapping. Accordingly, as compared to the $p(D)$ question, the $p(H \mid D)$ inference requires an added level of abstraction to notice that the relational role is not fixed and can vary with the form of the question.

The observed sample-type effect is also consistent with the relational-reasoning account, providing clear evidence that there is a cost when people are instructed to reason over a similar sample versus when the instructions direct reasoners to use the presented data. Of interest, however, the similarsample framing did not significantly impair reasoning with the 
$p(D)$ question in Study 2, and this was the case even with the added arithmetic step in this condition. This suggests that the misalignment between the presented and requested relations is the primary relational burden for Bayesian inference, which is made more complex by the added processing demands of the similar-sample question.

Error analyses revealed that the poorer performance in the similar-sample condition did not stem from a shift in strategy, but rather from confusion in the text comprehension process and/or from processing interference during mapping of the presented to the similar sample. Analysis of the "other" responses argued against the possibility that reasoners were using estimation strategies or computing the Bayesian response and then adjusting slightly for possible uncertainties in the newly imagined sample (a compute-then-adjust strategy). The fact that virtually all participants answered $p(D)$ with exactly " 40 out of 100 " also demonstrates that similar-sample questions do not inherently invoke estimation or computethen-adjust strategies. It might also be suggested that the prevalence of the superordinate value 100 indicated that participants were attempting to normalize responses. A careful review of protocols, however, argued against this possibility, with most of these responses showing drawn arrows or circles around the " 100 " in the text, or a simple summing of $80+20$, rather than normalization procedures. Furthermore, the 100 in the denominator was roughly equally paired with numbers presented in or directly derived from the text $(16,20$, or 40$)$, making the normalization explanation or the estimation strategy less likely. Although these patterns indicate that the error variability did not result from the application of estimation strategies or normalization procedures, future studies could look to more carefully specify the type of processing burden driving this effect.

As predicted, participants made fewer overall errors with the presented-sample scenarios, most of which resulted from using the hit rate, or the inverse fallacy (Villejoubert \& Mandel, 2002). The hit rate might be explained by superficial strategies such as label matching (Evans, 1998) or, more specifically, by confusing the structural roles (reference class and focal subset) during the mapping between the presented and requested relations (Holyoak \& Koh, 1987). That is, participants might make a less effortful direct mapping between the elements in the question and the presented text (infectedpositive) rather than a more demanding mapping requiring consideration of the relevant relational roles (total positive $\rightarrow$ infected). Hence, the more straightforward relational mapping promoted by the presented-sample scenario can explain both the increment of correct responses and the most frequent error found in this scenario.

Much work has gone into understanding why natural frequencies facilitate Bayesian performance. We were motivated by the general claims and empirical support for the nested-sets theory to further explore why natural frequencies still remain so difficult for so many reasoners. Previous work has looked at both the phrasing of the presented information (e.g., Krynski \& Tenenbaum, 2007; McNair \& Feeney, 2015) and the form of the question (e.g., Girotto \& Gonzalez, 2001; Pighin, Gonzalez, Savadori, \& Girotto, 2015), but no study has specifically looked at the role-based structural compatibility explored in the present study. One recent proposal by Ayal and Beyth-Marom (2014) looked at the "compatibility" of the presented and requested relations; however, their compatibility manipulations looked specifically at numerical aspects (formats and sample sizes), not at structural compatibility. They also reported an effect of "mental steps," referring to the number of explicit calculations needed to solve the problem. In our work, $p(D)$ and $p(H \mid D)$ required the same number of numerical steps, and therefore our alignment effect demonstrates an additional burden not tied to explicit numerical transformations - namely, the relational reasoning required to map the misaligned structures. We believe the naturalfrequency format enhances relational reasoning (see also DeWolf, Bassok, \& Holyoak, 2015). However, we also make the stronger claim that problems using percentages could also be viewed as relational-reasoning tasks requiring the alignment of role-based structured relations, in addition to the corresponding numerical transformations. Accordingly, the relational-reasoning framework predicts that similar alignment effects should be observable in Bayesian problems using percentage formats. Future studies could test this hypothesis.

To conclude, although natural frequencies have been hailed as a facilitator of Bayesian inferences for 20 years now (Gigerenzer \& Hoffrage, 1995), the fact that performance on these problems still remains so low has been little discussed. The present studies offer an explanation for this difficulty, by viewing Bayesian word problems as a case of relational reasoning, which requires the comparison of structural relationships across different levels of abstraction.

Author Note This research was supported by grants from the Generalitat de Catalunya (No. FI-DGR 2011) and the Spanish Ministry of Economics and Competitiveness (No. PSI2012-35703).

\section{References}

Ayal, S., \& Beyth-Marom, R. (2014). The effects of mental steps and compatibility on Bayesian reasoning. Judgment and Decision Making, 9, 226-242.

Barbey, A. K., \& Sloman, S. A. (2007). Base-rate respect: From ecological rationality to dual processes. Behavioral and Brain Sciences, 30 , 241-297.

Brase, G. L., \& Hill, W. T. (2015). Good fences make for good neighbors but bad science: A review of what improves Bayesian reasoning and why. Frontiers in Psychology, 6, 340. doi:10.3389/fpsyg.2015.00340

DeWolf, M., Bassok, M., \& Holyoak, K. J. (2015). Conceptual structure and the procedural affordances of rational numbers: Relational 
reasoning with fractions and decimals. Journal of Experimental Psychology: General, 144, 127-150.

Evans, J. S. B. T. (1998). Matching bias in conditional reasoning: Do we understand it after 25 years? Thinking \& Reasoning, 4, 45-82.

Evans, J. S. B. T., Handley, S. J., Perham, N., Over, D. E., \& Thompson, V. A. (2000). Frequency versus probability formats in statistical word problems. Cognition, 77, 197-213. doi:10.1016/S0010-0277 (00)00098-6

Gentner, D., \& Markman, A. B. (1997). Structure mapping in analogy and similarity. American Psychologist, 52, 45-56. doi:10.1037 /0003-066X.52.1.45

Gigerenzer, G., \& Hoffrage, U. (1995). How to improve Bayesian reasoning without instruction: Frequency formats. Psychological Review, 102, 684-704. doi:10.1037/0033-295X.102.4.684

Girotto, V., \& Gonzalez, M. (2001). Solving probabilistic and statistical problems: A matter of information structure and question form. Cognition, 78, 247-276.

Halford, G. S., Wilson, W. H., \& Phillips, S. (1998). Processing capacity defined by relational complexity: Implications for comparative, developmental, and cognitive psychology. Behavioral and Brain Sciences, 21, 803-865.

Halford, G. S., Wilson, W. H., \& Phillips, S. (2010). Relational knowledge: The foundation of higher cognition. Trends in Cognitive Sciences, 14, 497-505. doi:10.1016/j.tics.2010.08.005

Holyoak, K. J. (2012). Analogy and relational reasoning. In K. J. Holyoak \& R. G. Morrison (Eds.), The Oxford handbook of thinking and reasoning (pp. 234-259). New York, NY: Oxford University Press.

Holyoak, K. J., \& Koh, K. (1987). Surface and structural similarity in analogical transfer. Memory \& Cognition, 15, 332-340.

Johnson, E. D., \& Tubau, E. (2013). Words, numbers, and numeracy: Diminishing individual differences in Bayesian reasoning. Learning and Individual Differences, 28, 34-40. doi:10.1016/j. lindif.2013.09.004
Johnson, E. D., \& Tubau, E. (2015). Comprehension and computation in Bayesian problem solving. Frontiers in Psychology, 6, 938. doi:10.3389/fpsyg.2015.00938

Krynski, T. R., \& Tenenbaum, J. B. (2007). The role of causality in judgment under uncertainty. Journal of Experimental Psychology: General, 136, 430-450. doi:10.1037/0096-3445.136.3.430

Lesage, E., Navarrete, G., \& De Neys, W. (2013). Evolutionary modules and Bayesian facilitation: The role of general cognitive resources. Thinking \& Reasoning, 19, 27-53. doi:10.1080/13546783.2012.713177

Lipkus, I. M., Samsa, G., \& Rimer, B. K. (2001). General performance on a numeracy scale among highly educated samples. Medical Decision Making, 21, 37-44.

Mandel, D. R. (2007). Nested sets theory, full stop: Explaining performance on Bayesian inference tasks without dual-systems assumptions. Behavioral and Brain Sciences, 30, 275-276.

McNair, S. J., \& Feeney, A. (2015). Whose statistical reasoning is facilitated by a causal structure intervention? Psychonomic Bulletin \& Review, 22, 258-264. doi:10.3758/s13423-014-0645-y

Pighin, S., Gonzalez, M., Savadori, L., \& Girotto, V. (2015). Improving public interpretation of probabilistic test results: Distributive evaluations. Medical Decision Making, 35, 12-15. doi:10.1177/0272989 X14536268

Sirota, M., Juanchich, M., \& Hagmayer, Y. (2014). Ecological rationality or nested sets? Individual differences in cognitive processing predict Bayesian reasoning. Psychonomic Bulletin \& Review, 21, 198-204. doi:10.3758/s13423-013-0464-6

Sirota, M., Kostovičová, L., \& Vallée-Tourangeau, F. (2015). Now you Bayes, now you don't: Effects of set-problem and frequency-format mental representations on statistical reasoning. Psychonomic Bulletin \& Review, 22, 1465-1473. doi:10.3758/s13423-015-0810-y

Villejoubert, G., \& Mandel, D. R. (2002). The inverse fallacy: An account of deviations from Bayes's theorem and the additivity principle. Memory \& Cognition, 30, 171-178. 